\title{
Cosmetic use of botulinum toxin-A affects processing of emotional language
}

\author{
David A. Havas ${ }^{a}$, Arthur M. Glenberg ${ }^{a, b}$, Karol A. Gutowskic,d, Mark J. Lucarellic, and \\ Richard J. Davidson ${ }^{a}$ \\ a Department of Psychology, University of Wisconsin-Madison \\ b Department of Psychology, Arizona State University \\ c School of Medicine, University of Wisconsin-Madison \\ d Pritzker School of Medicine, University of Chicago
}

\begin{abstract}
How does language reliably evoke emotion, as when reading a favorite novel or listening to a skilled orator? Recent evidence suggests that comprehension involves a mental simulation of sentence content that calls on the same neural systems used in literal action, perception, and emotion. Here we demonstrate a causal role of involuntary facial expression in the processing of emotional language. Subcutaneous injections of botulinum toxin-A (BTX) were used to temporarily paralyze the facial muscle used in frowning. We found that BTX selectively slowed the reading of sentences with content that normally requires the paralyzed muscle for expression of a congruent emotion. This finding demonstrates a role of peripheral feedback in language processing, supports facial feedback theories of emotional cognition, and raises questions about effects of Botox on cognition and emotional reactivity. We account for the role of facial feedback in language processing by considering neurophysiological mechanisms and reinforcement learning theory.
\end{abstract}

Is language comprehension the manipulation of abstract symbols by rules of syntax (e.g., Chomsky, 1959)? Recent behavioral and neuroscientific evidence suggests that comprehension involves a mental simulation of sentence content (Barsalou, 1999) that calls on the same neural systems used in literal action, perception, and emotion (Glenberg \& Kaschak, 2002; Havas, Glenberg, \& Rinck, 2007; Niedenthal, 2007; Pulvermüller, 2005). Here we demonstrate a causal role of involuntary facial expression in processing of emotional language.

Havas et al. (2007) provide evidence for emotion simulation in language. Participants read and judged the valence of sentences describing pleasant situations ("You execute the difficult dive flawlessly") and unpleasant situations ("The police car pulls up behind you, siren blaring") while reading times were measured. Participants were instructed to hold a pen either in the teeth (to produce a smile) or in the lips (to prevent a smile) while reading. This procedure reliably induces emotional states in participants in the absence of awareness (Strack, Martin, \& Stepper, 1988). As predicted, reading times for sentences describing pleasant situations were faster while participants were smiling than while they were prevented from smiling, and the reverse was found for unpleasant sentences. Thus, facial

Coresponding author: David A. Havas, 1202 W. Johnson St., Madison, WI 53705; Phone: 608-262-1041, Fax: 608-262-4029, dahavas@wisc.edu. 
expressions influence simulation, but processes related to participants' voluntary control of facial posture could also provide a basis for the observed interactions.

As a prelude to the current research, previous work (e.g., Niedenthal, Winkielman, Mondillon, \& Vermeulen, 2009) has demonstrated that reading words describing emotions selectively activates facial muscles: Negative emotion words activate corrugator supercilii and positive emotion words activate zygomaticus. In addition, in an unreported experiment using the sentences described below, we verified that our happy sentences activated zygomaticus more than did sad and angry sentences, whereas sad and angry sentences more strongly activated corrugator supercilii than did happy sentences (see supporting information available on-line).

The question we tested here is whether this peripheral activation plays a causal role in sentence processing when voluntary control has been eliminated. Specifically, does paralysis of facial muscles used in expressing negative emotions (corrugator supercilii) selectively hinder processing of sentences describing angry and sad, relative to happy, situations? We recruited participants who were patients receiving cosmetic treatment of glabellar (frown) lines with subcutaneous injections of botulinum toxin type A (BTX). Participants were first time BTX patients, receiving injections only in the frown muscle (corrugator supercilii). BTX is a neurotoxin that causes temporary muscular denervation by preventing release of acetylcholine from presynaptic vesicles at the neuromuscular junction which in turn decreases extrafusal muscle fiber activity and muscle strength (Simpson, 1981). Toxicity is associated with a reduction in compound muscle action potential (CMAP) within 48 hours of intramuscular injection producing local muscle weakening within 1-3 days and peak weakening of CAMP around day 21 (Pestronk, Drachman, \& Griffin, 1976).

In two sessions (before and two weeks after BTX treatment), participants read sentences describing angry, happy, and sad situations and pressed a keyboard button when they understood the sentence. The dependent variable was sentence reading time. No reference to emotion was made during the task. By measuring change in reading times before and after BTX treatment, we were tested the prediction that paralysis of corrugator muscle selectively hinders processing of angry and sad, relative to happy, sentences.

\section{Method}

\section{Participants}

Forty-one female participants were recruited from among first-time patients receiving injections of botulinum toxin A (BTX) in corrugator supercilii muscle for treatment of glabellar (frown) lines. Patients were informed of the experiment through area cosmetic surgery clinics, and interested patients were scheduled for treatment during available experiment times. Participants in the study were given $\$ 50$ credit toward the cost of their treatment. Upon arrival at the clinic for the first session, all participants provided written informed consent for the reading experiment. One participant did not receive BTX injections and was excused from the study. The Education and Social and Behavioral Sciences Institutional Review Board at the University of Wisconsin-Madison approved the study protocol, and participants were treated in accordance with the principles expressed in the Declaration of Helsinki.

\section{Stimulus sentences}

In the first session, each participant read 20 Happy, 20 Sad, and 20 Angry sentences chosen at random from among 40 sentences of each valence (see Table 1). In the second session, the remaining 60 sentences were used. We verified sentence emotionality in a prior norming study, and for each sentence, we constructed one Yes/No comprehension questions for 
which the correct answer was "Yes," and one for which the correct answer was "No" (see supporting information available online).

\section{Procedure}

Sentences were presented on a laptop computer using E-Prime software (Psychology Software Tools, Inc., Pittsburgh, PA) in 5 blocks of 12 sentences, with each block separated by a brief pause for questions. Participants were instructed to press the "1" key on the keyboard number pad with the right index finger when they had finished reading a sentence. Reading time, the dependent variable of interest, was recorded for each sentence.

To encourage comprehension, a sentence was followed (after a one-second delay) by a Yes/ No comprehension question under the words "yes or no question" in a random third of the total number of trials in each block. Participants answered "Yes" by pressing the "X" key with the left index finger, or "No" with the "Z" key using the left middle finger. For the remaining trials, the sentence was replaced with a fixation cross for three seconds until the next sentence appeared.

At the clinic, participants entered a private room and signed a consent form. Participants received a) reading task instructions, b) five practice trials, and c) the 60 sentences. Upon completion, participants were scheduled for the second session, and immediately led to see the physician for BTX injection (see supporting information available online). Upon arrival at the clinic two weeks later for session 2, participants read the 60 remaining sentences, and then saw the physician for a check-up

The last 16 participants completed the 20-item PANAS-NOW self-report measure of positive and negative affect (Watson \& Clark, 1988) immediately upon completion of both first and second session reading tasks.

\section{Results}

Data were excluded from analysis for sentence reading times longer than 20 seconds and for one participant with an average sentence reading time greater than $9000 \mathrm{~ms}$. The overall accuracy rate for comprehension questions was $89 \%$. Each participant's reading times were subjected to a regression analysis using sentence length as a predictor. Trials greater than 2.5 standard deviations from their condition mean residual reading time were eliminated (removing $2.6 \%$ of trials).

A series of 3(sentence emotion: Angry, Sad, Happy) X 2(session: pre-injection, postinjection) repeated-measures ANOVAs were conducted on reading times, residual reading times, and comprehension accuracy rates. There were no significant effects in comprehension accuracy (all $F s<3.1$; see online supporting information). In reading times, there was a main effect of sentence emotion, where reading times were longer for Angry sentences $(M=4809 \mathrm{msec}, S E=187)$ than for $\operatorname{Sad}(M=4181 \mathrm{msec}, S E=170)$ or Happy sentences $(M=4020 \mathrm{msec}, S E=157), F(2,74)=128.798, p<.001, \eta^{2}=.392$. In addition, there was a significant interaction of sentence emotion and session as depicted in Figure 1, $F(2,74)=3.253, p=.044, \eta^{2}=.010$. Angry sentence reading times were significantly longer in the second session than in the first session, $t(37)=2.332, p=.025, d=.22$, as was the case with Sad sentences, $t(37)=2.348, p<.024, d=.23$. No changes were observed in Happy sentence reading times between first session and second session, $t(37)=.223, p=$. 825. No other effects were significant. Exactly the same pattern of significant effects was found in analyses of residual reading times, as depicted in Figure 2 (see supporting information available online). Analysis of mood measures during both sessions ruled out an 
explanation of the results based on mood congruency, such as a decrease in anxiety (see supporting information available online).

\section{Discussion}

The results demonstrate that blocking facial expression by peripheral denervation of facial musculature selectively hinders emotional language processing. This finding is consistent with embodied simulation accounts of cognition (Gallese, 2003; Gallese, 2008; Havas, et al., 2007) in which neural systems used in experiencing emotions are also used to understand emotions in language. The finding also offers evidence of a functional role for peripheral activation in processing emotional language, and suggests a bi-directional link between emotion and language mediated in part by moving the face. Finally, the finding provides novel evidence for facial feedback theories of emotion-related processing (Darwin, 1872/1998; Oberman, Winkielman, \& Ramachandran, 2007; Soussignan, 2002).

Although temporal order effects are a possibility in this study, a general order effect is unlikely given the selectivity of slow-down in reading times: the effect occurs only for angry and sad, but not happy, sentences. Also, the observed pattern is inconsistent with a regression to the mean, where angry and sad sentence reading times should be less extreme in the second session than in the first session. Next, we consider neurophysiological-level accounts of our data and then psychological-level accounts.

Given its known neurophysiological mechanisms of action, there are two principle ways BTX-induced paralysis of facial muscles might affect processing of emotional language. In both cases, we assume that the affected neural systems are recruited during a simulation of language content.

First, BTX may impair language processing through its peripheral muscle relaxant effects. By blocking cholinergic exocytosis at extrafusal neuromuscular junctions, BTX attenuates post-synaptic responses to voluntary and spontaneous motor efferent processes (Simpson, 1981). Neuromuscular blockade is established within 3-6 hours of treatment in rats (Pestronk, et al., 1976) although in humans, local muscle weakness is not observed sooner than 24 hours after injection for cosmetic treatment (Hamjian \& Walker, 1994).

Based on its initial peripheral effects, BTX could disrupt emotional processes that depend on stereotyped patterns of facial feedback (Soussignan, 2002). Alternatively, motor executive systems for controlling the affected muscles might respond to peripheral blockade with increased output but a loss of specificity as is seen in the cortical response to muscle fatigue (Gandevia, 2001). In either case, a loss of specificity in the mechanism for simulating angry and sad, but not happy, emotions would impair meaning resolution in processing of angry and sad, but not happy, sentences. A rapid mechanism is consistent with observations of immediate effects of facial posture manipulation (Havas, et al., 2007; Strack et al., 1988; Oberman, et al., 2007).

A related consideration is that BTX may have direct effects on the CNS via retrograde transport in afferent motoneurons. Evidence for direct effects on the CNS is weak, but a recent finding in rats showed that active BTX can be transported across afferent synapses and cause neurological changes remote from the injection site (Antonucci, Rossi, Gianfranceschi, Rossetto, \& Caleo, 2008). Here again, if successful execution of an efferent motor pattern (frowning) is necessary for simulation of angry and sad, but not happy, content, then retrograde denervation could conceivably interfere with processing angry and sad, but not happy, language. 
Second, BTX injections may affect language processing through central changes secondary to peripheral effects (Abbruzzese \& Berardelli, 2006). Studies in rats suggest that BTX affects cholinergic processing at intrafusal junctions, blocking gamma motor nerve endings and reducing tonic muscle spindle afferent discharge (Rosales, Arimura, Takenaga, \& Osame, 1996). In motor control, tonic afferent feedback is thought to contribute to formation of internal models of the state of the body, useful for specifying motor commands before a movement has begun. Patients with severe sensory neuropathy (a loss of afferent feedback) show systematic movement errors consistent with an inaccurate internal model of the state of the body (Ghez, Gordon, Gilardi, Christakos, \& Cooper, 1995).

Facial feedback may be a context-sensitive source of information for maintaining an internal model of the emotional state of the body, and important for specifying adaptive actions through its influence on central networks. Paralysis of corrugator muscle over a period of weeks might gradually induce plastic changes in neural circuitry underlying negative emotion state representation, leading to disrupted processing of angry and sad, but not happy, language. Consistent with this possibility, paralysis of corrugator muscle two weeks prior to an emotion expression imitation task reduced activation in neural centers involved in emotion processing, namely amygdala, orbitofrontal cortex, and brainstem centers involved in autonomic regulation, relative to activation in the same subjects before injection (Hennenlotter, et al., 2008) ${ }^{1}$. Whether these changes are due to early peripheral effects, or secondary central changes from BTX injection should be tested. In addition, our results suggest the need for further research on cognitive and emotional effects of cosmetic BTX injection.

At the psychological-level, our account of how feedback from emotional states guides simulation in language is suggested by a recent theory of reinforcement learning (Doya, $2007,2008)$. Because the most rewarding action in a situation depends on both the current state of the body and current state of the environment, choosing the most effective action in a situation is difficult. However, predicting the optimal action is possible if an agent can simulate the reward of a potential action given the current context. In reinforcement learning, the action value function is initially learned through feedback from the emotional state produced by a given action. Once this function has been learned, simulation of future actions will produce emotional state feedback useful for guiding subsequent action (Doya, 2008).

Consider reading the sentence, "Reeling from the fight with that stubborn bigot, you slam the car door." Comprehending words or phrases describing actions early in the sentence generates activity in the neural systems and periphery, including facial muscles, used in real actions or experiences (e.g., a fight with a stubborn person). Facial feedback would then contribute to autonomic and central changes (e.g., arousal) for preparing subsequent effective actions (e.g., slamming a car door). Because it is easier to simulate slamming a car door while the body is in an aroused state (Glenberg, Webster, Mouilso, Havas, \& Lindeman, 2009) the sentence is readily understood. Thus, to borrow the words of Darwin (1872/1998, p. 360-361), the free expression by outward signs of an emotion intensifies our processing of emotional language, whereas its repression hinders it.

\section{Supplementary Material}

Refer to Web version on PubMed Central for supplementary material.

\footnotetext{
${ }^{1}$ To the extent that these networks are involved in emotional experience, this mechanism might also play a role in the link between facial feedback and mood (Adelman \& Zajonc, 1989).
} 


\section{Acknowledgments}

We thank B. Webster, S. Mlinaz, A. Demet, L. Hazen, L. Greischar, C. van Reekum, and A. Buol for research assistance. Funding was provided by the National Science Foundation under Grants BCS-0315434 and BCS 0744105 to A. M. G., the National Institute of Mental Health under grants MH43454 and P50-MH084051 to R. J. D., and by an unrestricted grant from Research To Prevent Blindness, Inc. N.Y., N.Y. to the Department of Ophthalmology and Visual Sciences at the University of Wisconsin-Madison. Any opinions, findings, and conclusions or recommendations expressed in this material are those of the authors and do not necessarily reflect the views of the National Science Foundation or other funding agencies.

\section{Abbreviations}

TMS trans-cranial magnetic stimulation

MEP muscle-evoked potential

BTX botulinum toxin type-A

CMAP compound muscle action potential

\section{References}

Abbruzzese G, Berardelli A. Neurophysiological effects of botulinum toxin type A. Neurotoxicity Research. 2006; 9:109-114. [PubMed: 16785106]

Adelman PK, Zajonc RB. Facial efference and the experience of emotion. Annual Review of Psychology. 1989; 40:249-280.

Antonucci F, Rossi C, Gianfranceschi L, Rossetto O, Caleo M. Long-distance retrograde effects of botulinum neurotoxin A. Journal of Neuroscience. 2008; 28:3689-3696. [PubMed: 18385327]

Barsalou L. Perceptual symbol systems. Behavioral \& Brain Sciences. 1999; 22:577-660. [PubMed: 11301525]

Chomsky N. Review of B. F. Skinner's Verbal behavior. Language. 1959; 35:26-58.

Darwin, CR. The Expression of Emotions in Man and Animals. Oxford University Press; Oxford: 1872/1998.

Doya K. Reinforcement learning: Computational theory and biological mechanisms. HFSP Journal. 2007; 1:30. [PubMed: 19404458]

Doya K. Modulators of decision making. Nature Neuroscience. 2008; 11:410-416.

Gallese V. The manifold nature of interpersonal relations: the quest for a common mechanism. Philosophical Transactions of the Royal Society of London B: Biological Sciences. 2003; 358:517528.

Gallese V. Mirror neurons and the social nature of language: The neural exploitation hypothesis. Social Neuroscience. 2008; 3:317-333. [PubMed: 18979384]

Gandevia SC. Spinal and supraspinal factors in human muscle fatigue. Physiological Review. 2001; $81: 1725-1789$.

Ghez C, Gordon J, Gilardi MF, Christakos CN, Cooper SE. Impairments of reaching movements in patients without proprioception. II. Effects of visual information on accuracy. Journal of Neurophysiology. 1995; 73:361-372. [PubMed: 7714578]

Glenberg AM, Kaschak MP. Grounding language in action. Psychonomic Bulletin \& Review. 2002; 9:558-565. [PubMed: 12412897]

Glenberg AM, Webster BJ, Mouilso E, Havas D, Lindeman LM. Gender, emotion, and the embodiment of language comprehension. Emotion Review. 2009; 1:151-161.

Hamjian JA, Walker FO. Serial neurophysiological studies of intramuscular botulinum-A toxin in humans. Muscle \& Nerve. 1994; 17:1385-1392. [PubMed: 7969239]

Havas DA, Glenberg AM, Rinck M. Emotion simulation during language comprehension. Psychonomic Bulletin \& Review. 2007; 14:436-441. [PubMed: 17874584] 
Hennenlotter A, et al. The link between facial feedback and neural activity within central circuitries of emotion - New insights from Botulinum Toxin-induced denervation of frown muscles. Cerebral Cortex. June 17.2008

Niedenthal PM. Embodying emotion. Science. 2007; 316:1002-1005. [PubMed: 17510358]

Niedenthal PM, Winkielman P, Mondillon L, Vermeulen N. Embodiment of emotion concepts. Journal of Personality and Social Psychology. 2009; 96:1120-1136. [PubMed: 19469591]

Oberman LM, Winkielman P, Ramachandran VS. Face to face: blocking facial mimicry can selectively impair recognition of emotional expressions. Social Neuroscience. 2007; 2:167-178. [PubMed: 18633815]

Pestronk A, Drachman DB, Griffin JW. Effect of botulinum toxin on trophic regulation of acetycholine receptors. Nature. 1976; 264:787-789. [PubMed: 64930]

Pulvermüller F. Brain mechanisms linking language and action. Nature Reviews Neuroscience. 2005; 6:576-582.

Rosales RL, Arimura K, Takenaga S, Osame M. Extrafusal and intrafusal muscle effects in experimental botulinum toxin-A injection. Muscle \& Nerve. 1996; 19:488-496. [PubMed: 8622728]

Simpson LL. The origin, structure, and pharmacological activity of botulinum toxin. Pharmacological Review. 1981; 33:155-188.

Soussignan R. Duchenne smile, emotional experience, and autonomic reactivity: A test of the facial feedback hypothesis. Emotion. 2002; 2:52-74. [PubMed: 12899366]

Strack F, Martin LL, Stepper S. Inhibiting and facilitating conditions of the human smile: a nonobtrusive test of the facial feedback hypothesis. Journal of Personality and Social Psychology. 1988; 54:768-777. [PubMed: 3379579]

Watson D, Clark LA. Development and validation of brief measures of positive and negative effect: The PANAS Scales. Journal of Personality and Social Psychology. 1988; 54:1063-1070. [PubMed: 3397865] 


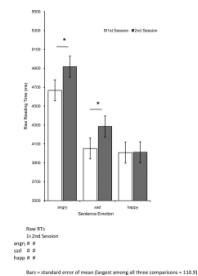

Fig. 1.

Emotional sentence (angry, sad, and happy) reading times (in Milliseconds) before ( $1^{\text {st }}$ session) and after ( $2^{\text {nd }}$ session) BTX injection in corrugator muscle. Error bars indicate $+/-1$ s.e.m. Significant comparisons are indicated with an asterisk. 


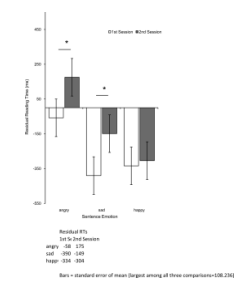

Fig. 2.

Emotional sentence (angry, sad, and happy) residual reading times (controlling for sentence length, in milliseconds) before ( $1^{\text {st }}$ session) and after ( $2^{\text {nd }}$ session) BTX injection in corrugator muscle. Error bars indicate $+/-1$ s.e.m. Significant comparisons are indicated with an asterisk. 


\title{
Table 1
}

Examples of Angry, Happy and Sad Sentences used in the experiment.

\author{
Angry sentences \\ Reeling from the fight with that stubborn bigot, you slam the car door. \\ The pushy telemarketer won't let you return to your dinner. \\ The workload from your pompous professor is unreasonable. \\ Happy sentences \\ The water park is refreshing on the hot summer day. \\ Finally, you reach the summit of the tall mountain. \\ You spring up the stairs to your lover's apartment. \\ Sad sentences \\ You hold back your tears as you enter the funeral home. \\ You open your email in-box on your birthday to find no new emails. \\ Your closest friend has just been hospitalized for a mental illness.
}

